\title{
Study on the Hydrodynamic Performance of a Floating Kuroshio Current Turbine
}

\author{
Jing-Fa Tsai, Yi-Xuan Zeng, Forng-Chen Chiu \\ Department of Engineering Science and Ocean Engineering, National Taiwan University \\ No. 1 Roosevelt Road, Taipei, Taiwan \\ jftsai@ntu.edu.tw; r03525101@ntu.edu.tw;fcchiu@ntu.edu.tw
}

\begin{abstract}
A 20KW prototype floating current turbine was designed for operating in the Kuroshio Current, passes along the eastern coast of Taiwan at a maximum speed of approximately $1.5 \mathrm{~m} / \mathrm{s}$. The location and speed of the Kuroshio Current are generally consistent, offering Taiwan a stable and secure energy source. A test apparatus, which included a 800-W 1/5 scale model of the proposed turbine, was prepared to measure the rotation, thrust and torque of the designed turbine. The apparatus was tested in a towing tank at constant speed. The rotation of the turbine was controlled using an electronic brake to simulate the torque induced by the generator. The power coefficient, torque coefficient and thrust coefficient were calculated from the measured data. The relationships of these three coefficients with tip speed ratio were determined. The measured power coefficient and torque coefficient agreed well with the calculated results. Whereas the measured thrust coefficient was higher than that calculated. The likely cause of the high measured thrust coefficient is discussed in this paper.
\end{abstract}

Keywords: Floating Kuroshio Current Turbine, Hydrodynamic Performance, Towing Tank.

\section{Introduction}

Global warming is caused by the emission of greenhouse gases, which are generated by the burning of fossil fuels. To address the critical issue of global warming, the United Nations Framework Convention on Climate Change established the Kyoto Protocol[1], the Copenhagen Accord[2] and the COP21[3] to curb climate change and limit the emission of greenhouse gases. To reduce the greenhouse gas emissions, alternative energy sources that are renewable and eco-friendly are required. Numerous types of energy can be sourced from the ocean, such as wind, wave, tidal, current and ocean thermal energy. Wind power is a mature technology that is well established on land. Offshore wind farming is also under development, and has the potential to become a major contributor to the electric energy market [4]. However, technologies to harvest other forms of ocean energy, such as tidal, wave and current, are still in their infancy. Stability is often a maj or challenge with renewable energy. For example, tidal current occurs once or twice daily and persist for inconsistent periods of time. Wave depends on weather. By contrast, ocean currents, continuous directed movements of seawater, are more stable and are the most dependable form of ocean energy. Currents flow for great distances and play a dominant role in determining the climate of many regions. The Kuroshio Current is a north-flowing ocean current on the west side of the North Pacific Ocean. It begins off the east coast of Luzon, Philippines, passes along the coast of Taiwan and Japan as it flows northeast, where it merges with the easterly drift of the North Pacific Current. The Kuroshio Current is the most important current in the seas east of Taiwan. It is stable and carries a large amount of ocean energy. Hydrographic surveys[5] have revealed that the distance of the high-velocity core from the coast of Taiwan at $23.75 \mathrm{~N}$ is $30-120 \mathrm{~km}$ with maximum current speeds 0.60-1.20 m/s. Transport speed varied between 15 and $26 \mathrm{~Sv}(1 \mathrm{~Sv}=106 \mathrm{~m} 3 / \mathrm{s})$ in studies after 1990[5].

Current energy is of two types: tidal current and ocean current. Tidal currents are created by gravitation pull. The SeaGen marine current turbine (MCT)[6] was the first successful tidal current turbine. Ocean currents are a continuous, directed movement of seawater generated by various forces, such as wind, the Coriolis force and differences of temperature and salinity [7]. Ocean currents represent an uninterrupted flow of green energy. Their power is in the form of kinetic energy. It is known that they are more predictable than wind and solar power. In addition, they are stable and steady when compared to other types of ocean energy. To utilize current energy, Batten et al.[8] proposed a method to design current turbine and conducted a performance test of the pitch angle effect in a cavitation tunnel. Bahaj et al.[9] studied the effect of 
yaw on the performance of a current turbine both in a cavitation tank and a towing tank. Batten et al.[10] also developed and verified a blade element method to predict the hydrodynamic performance of a single current turbine. The IHI corporation [11] proposed a floating counter-rotating twin-blades turbine to counterbalance rotatory torque. Shirasawa et al.[12] also designed an ocean current turbine for operation in the Kuroshio Current. The Aquantis Current Plane (" $\mathrm{C}$ Plane") technology [13] was developed for power generation in the Gulf Stream off of Florida, USA. In addition, The Center for Ocean Energy Technology of Florida Atlantic University developed a single-turbine system. It is tethered to a buoy which is permanently moored. The Gulf stream turbine system developed by Robson[14-15] for the Gulf Stream Current is a more complicated one. A self-supporting structure was design that allowed the turbines to be positioned at depths for which the current could generate electricity at about the design capacities of the turbine.

In this study, a floating marine current turbine is proposed and designed for operation in the Kuroshio Current. An apparatus is devised to measure the hydrodynamic performance of the designed current turbine model and to verify the design target.

\section{Proposed Kuroshio Current Turbine}

A $20 \mathrm{KW}$ watts counter-rotating twin turbine current turbine was proposed for the Kuroshio Current turbine project as shown in Fig. 1. The power $20 \mathrm{KW}$ is estimated assuming a power coefficient of 0.3 . The diameter of the turbine is $5 \mathrm{~m}$ and the inflow velocity is $1.5 \mathrm{~m} / \mathrm{s}$. The counter-rotating twin turbines were designed to reduce the torque imbalance of the rotating blades. Each turbine holds three blades and produces $10 \mathrm{~kW}$ of energy. The proposed turbine was designed to be placed downwind to minimize yawing instability. The foil type float was designed to control the turbine attitude during launch and retrieval. Table 1 lists the dimensions of the proposed turbine. The section of the blade is a NACA 66 a=0.8 meanline.

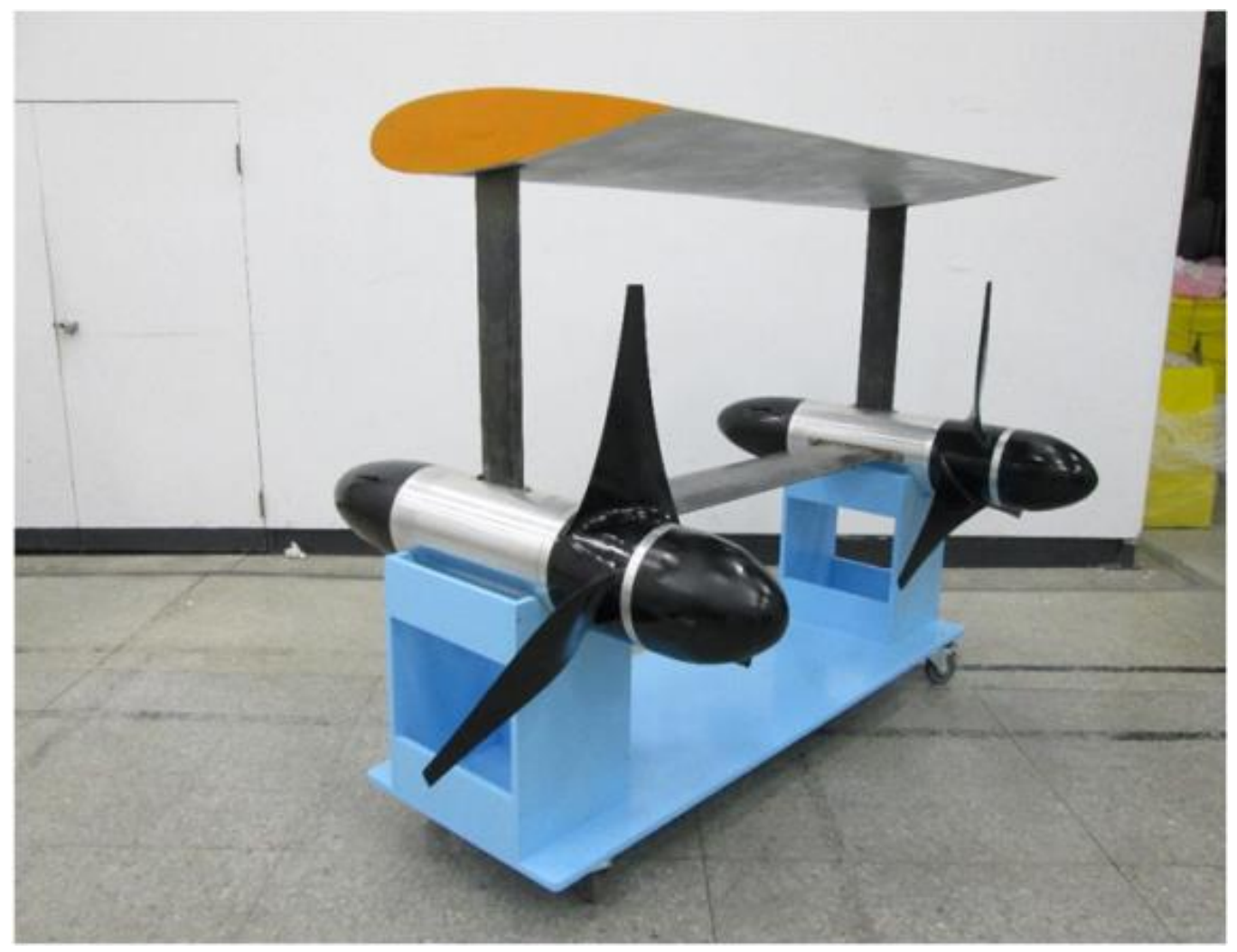

Fig. 1: Proposed Kuroshio Current turbine. 
Table 1: Dimensions of the Prototype Kuroshio Current Turbine.

\begin{tabular}{|c|c|}
\hline Item & Specification \\
\hline Blade diameter & 5 meters \\
\hline Blade section & NACA $66 \mathrm{a}=0.8$ \\
\hline Floater chord length & 4 meters \\
\hline Floater span & 8 meters \\
\hline Nacelle diameter & 1.2 meters \\
\hline Nacelle length & 6 meters \\
\hline $\begin{array}{c}\text { Distance between } \\
\text { blade center }\end{array}$ & 7.5 meters \\
\hline
\end{tabular}

\section{Hydrodynamic Performance Coefficients of a Current Turbine}

The hydrodynamic performance of a current turbine can be assessed by examining the relationships of the power coefficient, torque coefficient and thrust coefficient with the tip speed ratio of the turbine. The power coefficient is defined as

$$
C_{p}=\frac{P}{\frac{1}{2} \rho U_{0}^{3} A}
$$

where $\mathrm{P}$ is the power generated by the turbine, $U_{0}$ is the inflow velocity, and $\mathrm{A}$ is the section area of the turbine. The torque coefficient is defined as

$$
C_{Q}=\frac{Q}{\frac{1}{2} \rho U_{0}^{2} A}
$$

where $\mathrm{Q}$ is the torque generated by the turbine. The thrust coefficient is

$$
C_{T}=\frac{T}{\frac{1}{2} \rho U_{0}^{2} A}
$$

where $\mathrm{T}$ is the thrust produced by the turbine. The tip speed ratio is

$$
\lambda=\frac{\varpi R}{U_{0}}
$$

where $\omega$ and $R$ are the rotation speed and radius of the turbine, respectively.

The hydrodynamic performance of the current turbine can be calculated using software or can be determined using model test. A $1 / 5$ scale model turbine with a diameter of $1 \mathrm{~m}$ was manufactured and subjected an open water test in a towing tank at National Taiwan university for measuring the towing speed, revolution per second of the turbine, thrust and torque. Using these measurements, the power coefficient, torque coefficient and thrust coefficient can be calculated.

\section{Test Apparatus and Model Turbine}

As shown in Fig. 2, a dynamometer that can measure the rotation, thrust and torque was designed and manufactured to measure the hydrodynamic performance of the current turbine. An electromagnetic brake was coupled with the dynamometer to simulate the generator torque payload. A photoelectric tachometer of 60 pulses per rotation was installed to measure the rotation of the current turbine. The capacity of the thrust and torque meters are $100 \mathrm{~kg}$ and $400 \mathrm{~kg}-\mathrm{cm}$, respectively, and Fig. 3 and 4 show the corresponding calibration curves. The entire system was installed within the nacelle 
of the turbine. The test apparatus with the model turbine was attached on a foil support and subjected to a test $2 \mathrm{~m}$ underwater in the towing tank. Fig. 5 shows the entire 1/5 scale model turbine with a diameter of $1 \mathrm{~m}$, foil float, vertical supports and horizontal connector between nacelles.

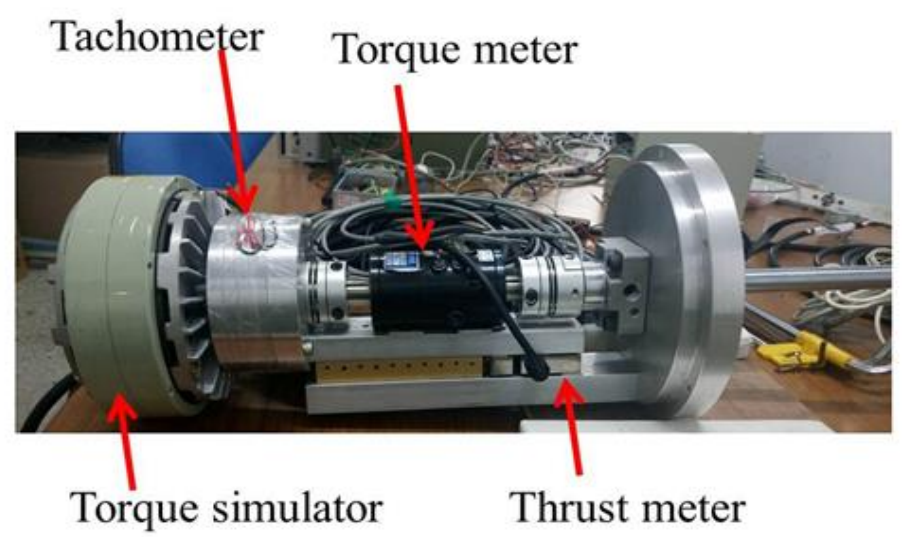

Fig. 2: Dynamometer of the current turbine.

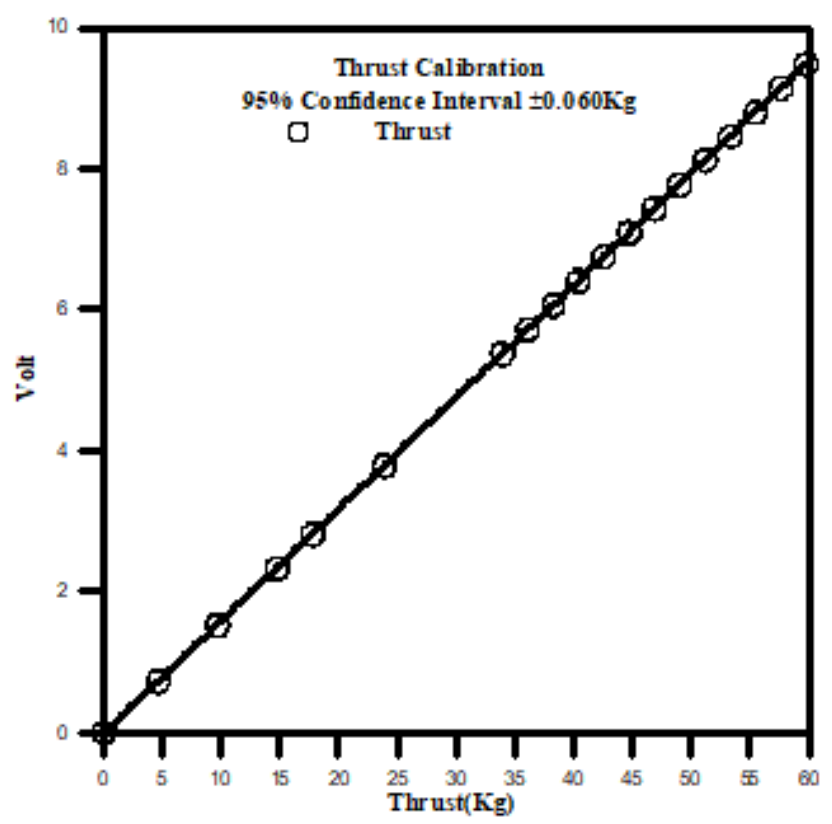

Fig. 3: Thrust calibration curve. 


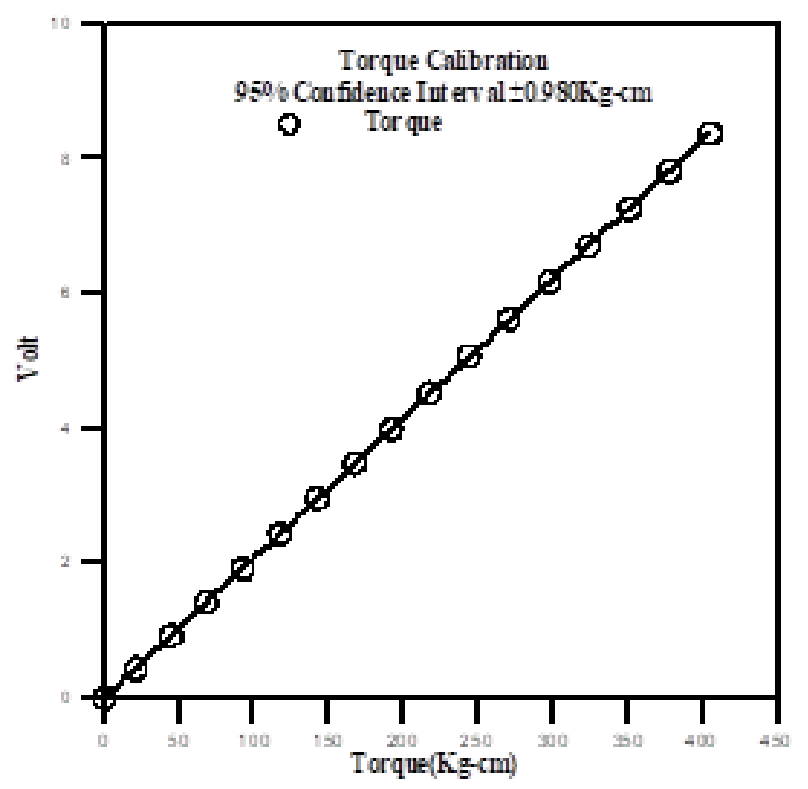

Fig. 4: Torque calibration curve.

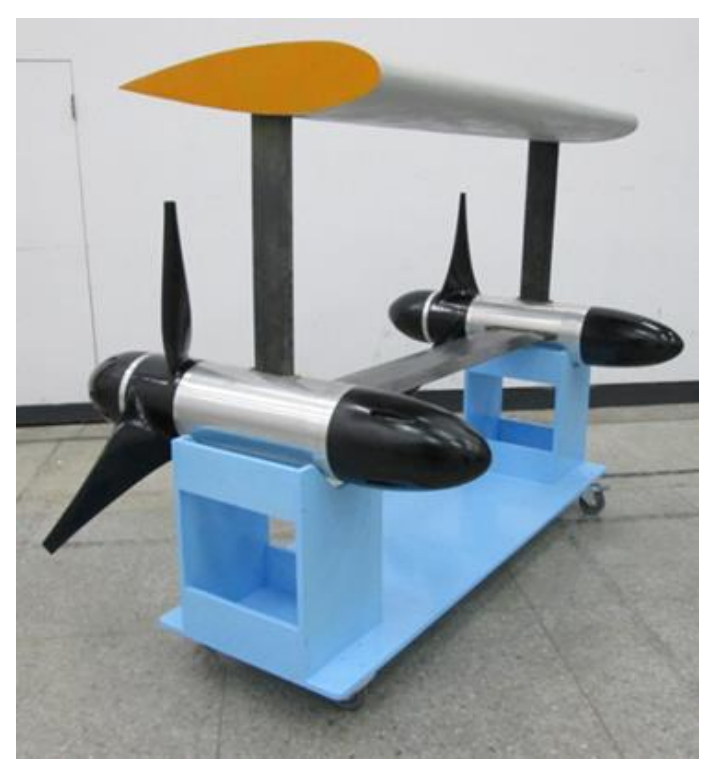

Fig. 5: Model turbine.

\section{Test Results and Discussions}

The towing speed was set to $1.0,1.2$ and $1.5 \mathrm{~m} / \mathrm{s}$ in this study. The torque payload was provided by the electrical brake. The rotation of the blade was controlled by the torque payload. The rotation, thrust, torque and towing speed were measured. Fig.6 and 7 show the measured power coefficient curve as a function of the tip speed ratio of the clockwise and counter-clockwise turbine. The measured power coefficients agreed well with the calculated result using FLUENT RANS code. Fig. 8 and 9 show the measured torque coefficient curve as a function of tip speed ratio of the clockwise and counterclockwise turbine s, respectively. The measured torque coefficients also agreed well with the calculated torque coefficient except when the value of tip speed ratio is smaller than 4 in the clockwise turbine. The measured torque coefficient is higher than those calculated. Fig. 10 and 11 show the measured thrust The measured thrust coefficients were higher than those calculated as shown in Fig.10 for clockwise turbine. Fig.11 shows the measured thrust coefficients were distributed widely around those calculated. However, most of the measured thrust coefficients were higher than the calculated coefficients, except at $1.0 \mathrm{~m} / \mathrm{s}$. The hydrodynamic performance of the current turbine is similar to that of a wind turbine. 


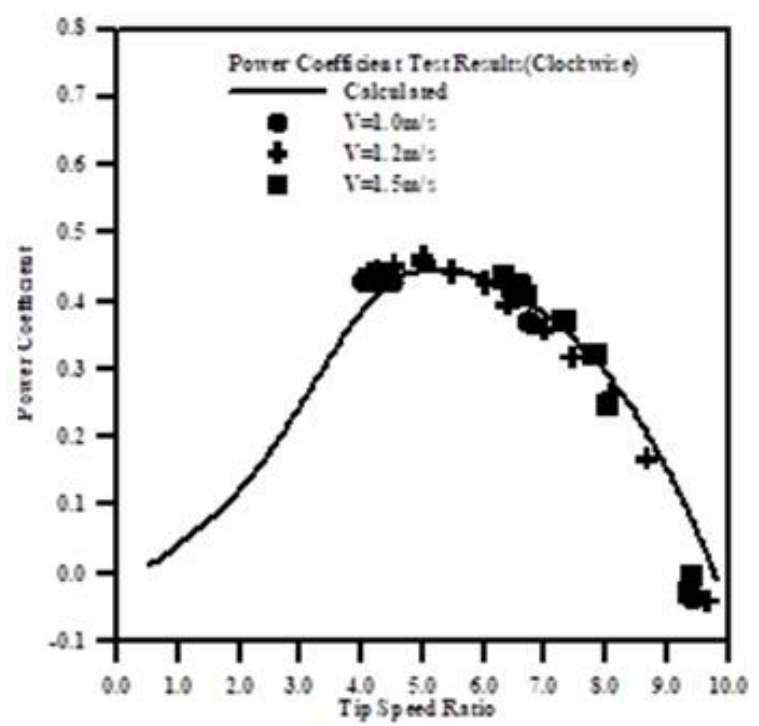

Fig. 6: Power coefficient of the clockwise turbine.

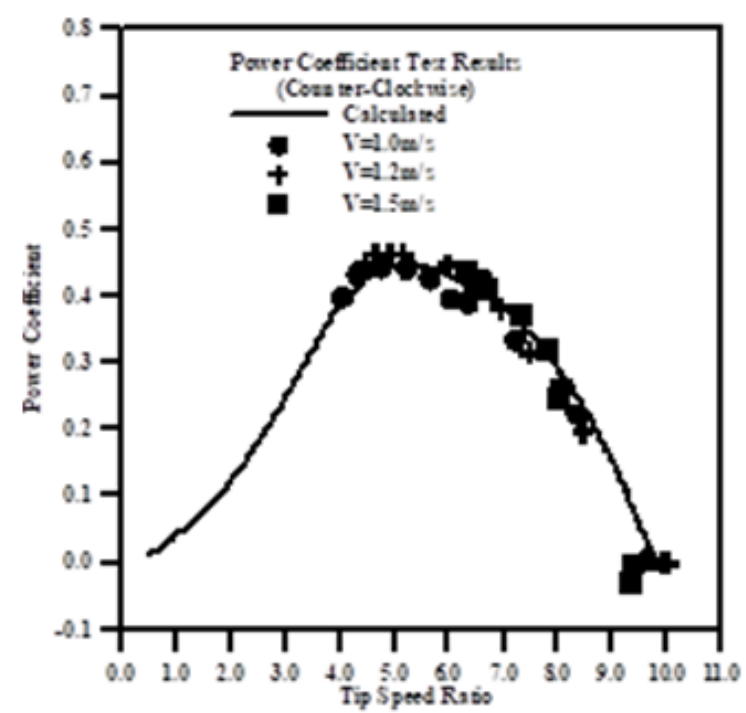

Fig. 7: Power Coefficient of the counter-clockwise turbine. 


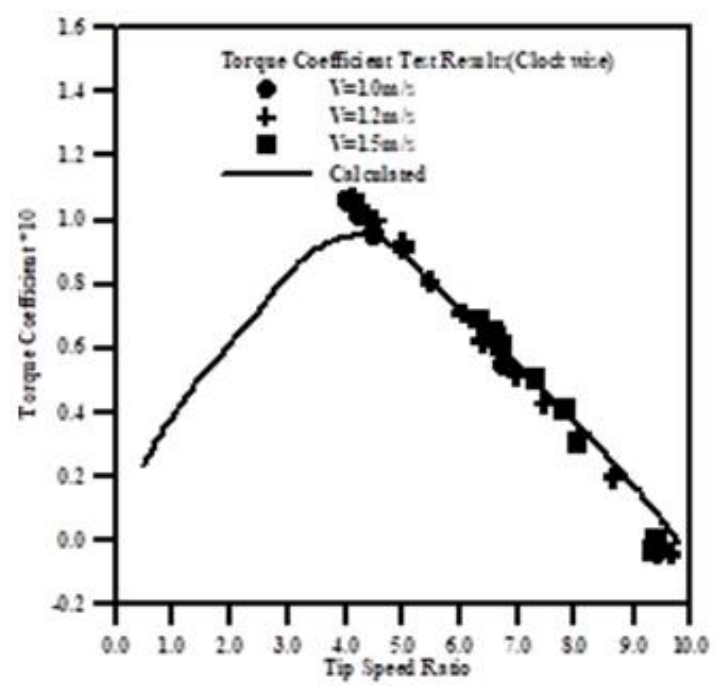

Fig. 8: Torque coefficient of the clockwise turbine.

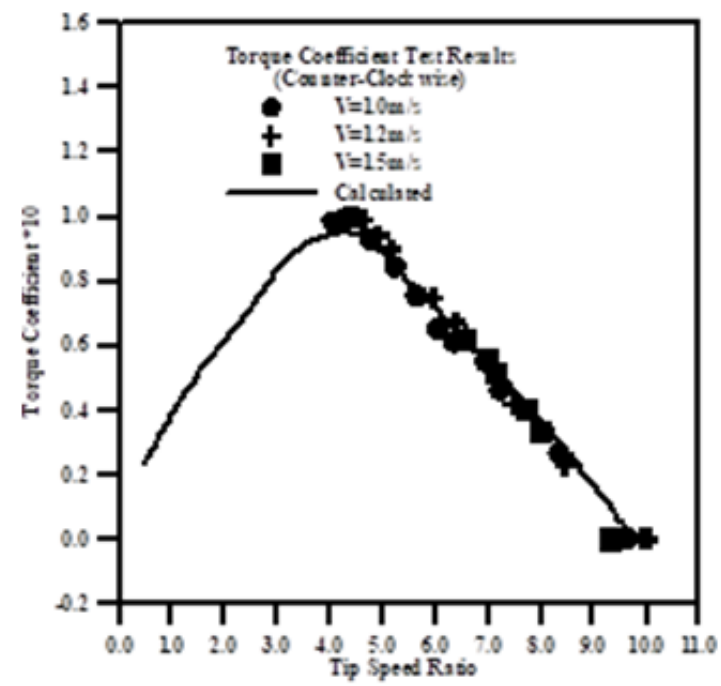

Fig. 9: Torque coefficient of the counter-clockwise turbine.

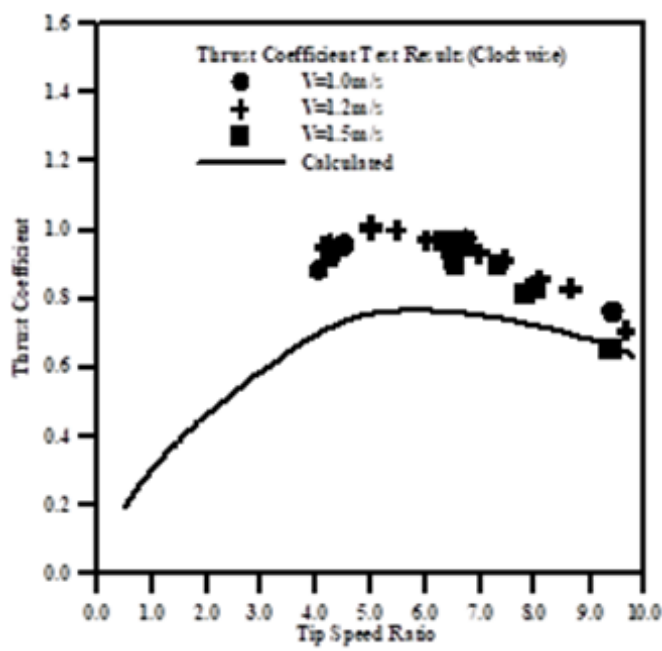

Fig. 10: Thrust coefficient of the clockwise turbine. 


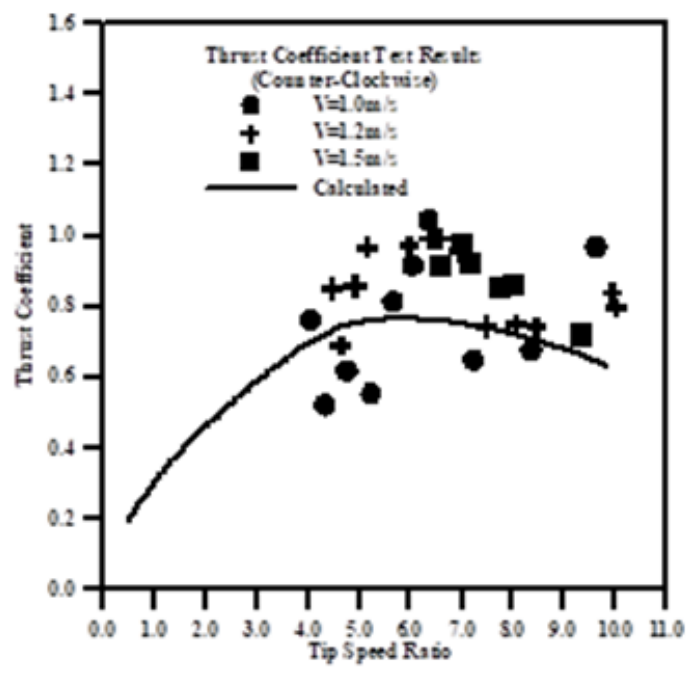

Fig. 11: Thrust coefficient of the counter-clockwise turbine.

In Fig.11, the measured thrust coefficients are distributed widely around calculated thrust curve. However, most of the measured thrust coefficients are higher than that of calculated except for that of the low speed $1.0 \mathrm{~m} / \mathrm{s}$. The hydrodynamic performance of the current turbine is similar with the wind turbine. For the study of wind turbine, most the measured thrust coefficients were also higher than the calculated thrust coefficient. Buhl[16] proposed a new empirical thrust curve with induction factor, which is higher than 0.5, according to the measured thrust coefficient of Lock[17]. The measured higher thrust coefficient is due to the turbulent state of the wake behind the turbine which makes the induction factor is higher than 0.5 as shown in Fig. 12. The measured high thrust coefficient is due to the turbulent wake state when the induction factor is greater than 0.5 .

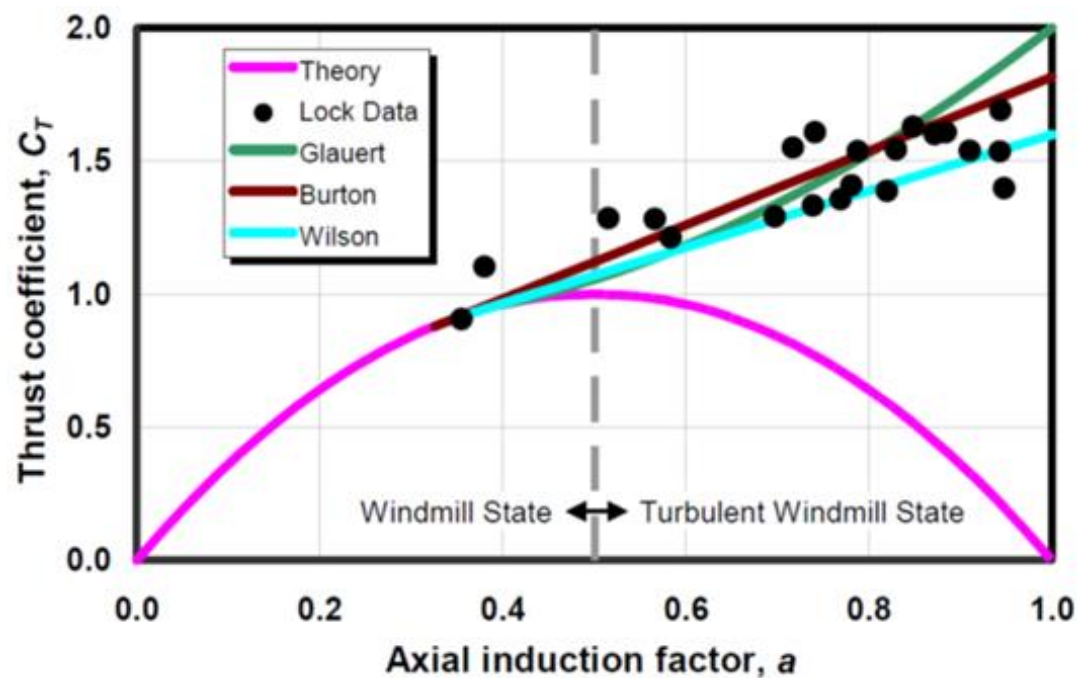

Fig. 12: The Thrust Coefficient with Induction Factor of Wind Turbine.

\section{Conclusion}

A floating twin-blade counter rotating current turbine was proposed and designed for operating in the Kuroshio Current. A 1/5 model turbine was manufactured and subjected to an open water test in a towing tank. The measured power coefficients and torque coefficients agreed well with the calculated power and torque coefficients. However, the measured thrust coefficients were higher than those calculated, likely because of the turbulent wake which increased the induction factor to more than 0.5. Future studies can address this discrepancy. 


\section{Acknowledgements}

The authors thank the Ministry of Science and Technology of the Republic of China for the financial support of the Floating Kuroshio Current Turbine Project. (MOST 104-3113-E-002-020-CC2).

\section{References}

[1] United Nations, Kyoto Protocol to the United Nations Framework Convention on Climate Change, unfccc.int/kyoto_protocol, 1998.

[2] United Nations, Copenhagen Accord Framework Convention on Climate Change, unfecc.int/resource/docs/2009/cop15/, 2009.

[3] United Nations, UNFCCC COP 21 Paris France - 2015 Paris Climate Conference, [Online]. Available: www.cop21 paris.org

[4] A. Smith, T. Stehly, and W. Musial, "2014-2015 Offshore Wind Technologies Market Report," Technical Report, NREL/TP-5000-64283, 2015.

[5] Y. C. Hsin, C. R. Wu, and P. T. Shaw, "Spatial and temporal variations of the Kuroshio east of Taiwan,1982-2005: A numerical study," Journal of Geophysical Research, vol. 113, 2008.

[6] P. L. Fraenkel, "Development and testing of Marine Current Turbine's SeaGen 1.2MW tidal stream turbine," 3rd International Conference on Ocean Energy, Bilbao, 2010.

[7] A. H. Kevin, M. F. Hermann, P. F. Steven and S. N. Vincent, "Assessment of Energy Production Potential from Ocean Currents along the United States Coastline," Georgia Tech Research Corporation, 2013.

[8] W. M. J. Batten, A. S. Bahaj, A. F. Molland and J. R. Chaplin, "Hydrodynamics of marine current turbines," Renewable Energy, vol. 31, pp. 249-256, 2006.

[9] A. S. Bahaja, A. F. Mollandb, J. R. Chaplina and W. M. J. Batten, "Power and thrust measurements of marine current turbines under various hydrodynamic flow conditions in a cavitation tunnel and a towing tank," Renewable Energy, vol. 32, pp. 407-426, 2007.

[10] W. M. J. Battena, A. S. Bahaja, A. F. Mollandb, and J. R. Chaplin, "The prediction of the hydrodynamic performance of marine current turbines," Renewable Energy, vol. 33, pp. 1085-1096, 2008.

[11] IHI Corporation, "Power Generation Using the Kuroshio Current," IHI Engineering Review, vol. 46, no. 2, pp. 1-5, 2014.

[12] K. Shirasawa, J. Minami, H. Iwashita and T. Shintake, "Development of a ocean-current turbine for the Kuroshio Current," 6th International Symposium on Energy Challenges and Mechanics toward a Big Picture, Inverness Scotland UK, 2016.

[13] A. Fleming, "Aquantis ocean current turbine development: innovative power generation technology," Dehlsen Associates, LLC, Santa Barbara, CA and US Department of Energy, Washington, DC, 2014.

[14] J. H. Robson, "Submersible electrical power generating plant," US Patent, No. 6531788, 2003.

[15] J. H. Robson, "Submersible electrical power generating plant," US Patent, No. 7691936, 2007.

[16] M. L. Buhl, Jr., "A New Empirical Relationship between Thrust Coefficient and Induction Factor for the Turbulent Windmill State," Technical Report, NREL/TP-500-36834, 2005.

[17] C. N. H. Lock, H. Batemen, H. C. H. Townsend, "An Extension of the Vortex Theory of Airscrews with Applications to Airscrews of Small Pitch, Including Experimental Results," No. 1014. Aeronautical Research Committee Reports and Memoranda, 1926. 